\title{
Aproximación a las actitudes y percepciones de los usuarios ante las tecnologías de la información
}

\author{
Por María-Dolores Olvera-Lobo y Belarmina Benítez-de-Vendrell
}

\begin{abstract}
Resumen: La comprensión de por qué la gente acepta o rechaza las tecnologías de la información ha demostrado ser una de las cuestiones más desafiantes de la investigación en nuestro ámbito. Con el fin de entender las opiniones individuales acerca de las tecnologías de la información y de la comunicación (TIC) algunos estudios tratan de identificar las percepciones de los usuarios en relación a los conceptos de eficacia, ansiedad, satisfacción, y utilidad, basándose en factores relacionados con la actitud. En este trabajo se ofrece una revisión de algunas de las principales aportaciones relacionadas con las actitudes y las percepciones de los usuarios ante las tecnologías de la información así como de algunos de los estudios que han aplicado estas metodologías de evaluación, tanto en el contexto general del uso de los ordenadores, como en el ámbito específico de la tecnología web. El análisis nos lleva a la conclusión de que no importa lo sofisticada y de cuánto es capaz la tecnología, su puesta en práctica eficaz depende de que los usuarios tengan una actitud positiva hacia ella.
\end{abstract}

Palabras clave: Actitudes hacia el ordenador, Usuarios de tecnologías de la información, Medidas de usuarios, Ansiedad hacia el ordenador, Percepciones de los usuarios.

Title: User attitudes and perceptions toward information technology

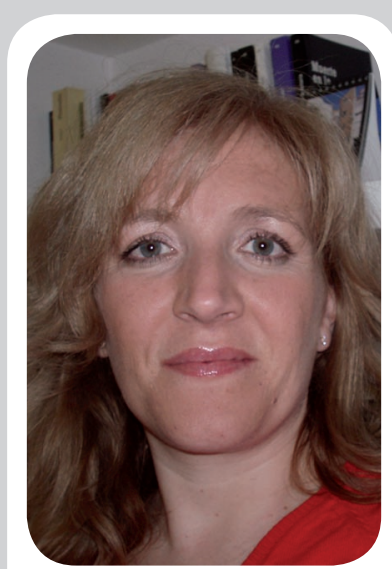

María-Dolores Olvera-Lobo es doctora en documentación, profesora titular del Departamento de Biblioteconomía y Documentación, docente en la Facultad de Comunicación y Documentación y en la Facultad de Traducción e Interpretación de la Universidad de Granada. Participa en varios proyectos de investigación y es autora de libros y artículos especializados.

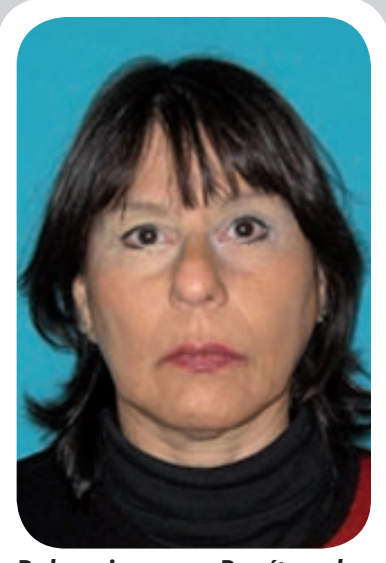

Belarmina Benítez-deVendrell es licenciada en bibliotecología y documentación, y profesora titular en la Universidad Nacional de Misiones, Argentina, donde dirige proyectos de investigación. Es autora de libros y artículos especializados en biblioteconomía y ciencias de la información y doctorando en documentación por la Universidad de Granada.

Abstract: Understanding why information technology is accepted or rejected by society has become one of the most challenging issues for researchers in our field. In order to understand personal attitudes toward communication and information technology (CIT), some researchers attempt to identify user perceptions of efficacy, anxiety, satisfaction, and usefulness, based on attitude factors. We discuss some of the primary research findings related to user attitudes and perceptions toward information technology. In addition, we review key studies that have implemented these assessment methods, within both the general context of computer use and the specific environment of web technology. We conclude that the level of technological sophistication and capacity are less relevant to effective implementation than positive user attitudes toward the technology.

Keywords: Computer attitudes, Information technologies users, User measurement, Computer anxiety, User perception.

Olvera-Lobo, María-Dolores; Benítez-de-Vendrell, Belarmina. "Aproximación a las actitudes y percepciones de los usuarios ante las tecnologías de la información”. En: El profesional de la información, 2008, marzo-abril, v. 17, n. 2, pp. 199-204.

DOI: $10.3145 /$ epi.2008.mar.10

\section{Introducción}

LA MASIFICACIÓN DEL USO DE INTERNET y de la web ha proporcionado oportunidades y desafíos sin precedentes en todas las áreas del saber y del quehacer humano. Con el fin de entender las opiniones individuales de los usuarios acerca de las tecnologías de la información y de la comunicación (TIC), diferentes estudios tratan de identificar las percepciones de éstos en relación a los conceptos de eficacia, ansiedad, satisfacción, y utilidad, basándose en factores relacionados con la actitud.

Algunas de estas propuestas son la Computer Attitude Scale de Loyd y Gressard (1984), la Attitu-
de-Toward-Computer Usage Scale de Popovich, Hyde y Zakrajsek (1987); o la Computer Attitude Measure de Kay (1993). La mayoría de estos instrumentos miden actitudes hacia el ordenador de una forma genérica, sin especificaciones concretas hacia aplicaciones particulares. Metodológicamente son estudios exploratorios en los que los datos se 
recaban mediante cuestionarios, o a través de entrevistas y/o discusión en grupo.

Entre los diversos modelos teóricos desarrollados para examinar las intenciones de los usuarios en relación al uso del ordenador y de las TIC, la propuesta del Modelo de Aceptación de la Tecnología -Tecnology Acceptance Model (TAM)ha destacado por ser especialmente prometedora. TAM (Davis, 1994) explica la aceptación de la tecnología basándose en las actitudes de los individuos. Está ligado a la teoría cognitiva social -Social Cognoscitive Theory (SCT)- (Bandura, 1986; Compeau; Higgins, 1995) por dos constructos dominantes que determinan el uso de la tecnología: la utilidad percibida (PU) y la facilidad de uso percibida (PEU).

La PU se define como la opinión subjetiva del usuario sobre el grado en el que el funcionamiento de un sistema informático o de un software ayudará al trabajo y por ende mejorará su desempeño. Por su parte, la PEU se refiere al grado en el cual el usuario espera que la utilización de un sistema informático o software no implique esfuerzo adicional. Por lo tanto, la PEU es utilizada como medida de las expectativas respecto al proceso y la PU como medida de las expectativas respecto al resultado o fruto de las acciones.

La investigación centrada en el modelo $T A M$ ha proporcionado aproximaciones respecto al uso de las TIC en general, pero se ha centrado en la PU como determinante del uso antes que en otros factores (Lederer, et al., 2000). TAM sugiere que los usuarios utilizan la informática si creen que dará lugar a resultados positivos. No obstante, una crítica a esta propuesta es que el modelo asume que cuando alguien se forma una intención de actuar lo hará libremente y sin limitaciones, pero sabemos que en el mundo real hay muchas restricciones -tales como una capacidad limitada, los apremios de tiempo, los límites del entorno o de la organización, o los hábitos inconscientes- que reducirán la libertad para actuar (Bagozzi; Davis; Warshaw., 1992).

A continuación detallamos algunos de los principales factores que influyen en las actitudes y percepciones de los usuarios respecto a la aceptación y uso de las TIC, a saber: la ansiedad, la eficacia, la satisfacción y la utilidad percibidas. Posteriormente se comentan algunas aproximaciones a la aceptación y uso de la tecnología web y por último se muestran unas reflexiones finales.

\section{Factores que influyen en las actitudes y percepciones de los usuarios}

La comprensión de por qué la gente acepta o rechaza las tecnologías de la información ha demostrado ser una de las cuestiones más desafiantes de la investigación en el ámbito de los sistemas de información. Hebert y Benbasat (1994) descubrieron que el $77 \%$ de la varianza en el intento de utilizar las tecnologías de la información se podría explicar por actitudes hacia los ordenadores.

\section{"La comprensión de por qué la gente acepta 0 rechaza las tecnologías de la información ha demostrado ser una de las cuestiones más desafiantes de la investigación en nuestro ámbito"}

La escala de actitud desarrollada por Loyd y Gressard incluye cuatro factores importantes: 1) la ansiedad percibida, es decir el miedo y la ansiedad hacia el ordenador o la tendencia de una persona a sentirse inquieto y aprensivo ante la idea de su uso presente o futuro; 2) la eficacia percibida o confianza, entendida como la capacidad de utilizar los ordenadores o de aprender sobre ellos; 3) la satisfacción percibida o complacencia al trabajar con los ordenadores y; 4)la utilidad percibida, que describe el grado de utilidad que se percibe al usar los ordenadores para el trabajo presente y futuro (Al-Khaldi; Al-Jabri, 1998).

En general la ansiedad, la eficacia y la satisfacción representan la parte afectiva de la actitud, dejando fuera el aspecto cognitivo de ésta (Liaw, 2002a, 2002b). De acuerdo con los conceptos del modelo de aceptación de la tecnología, la intención de utilizar la tecnología es el factor principal que nos permite entender las opiniones individuales respecto a la aceptación y uso de ésta (Vankatesh, 1999).

\section{Ansiedad percibida}

Entre las sensaciones negativas, la ansiedad se refiere a la tendencia de los individuos a ser tecnofóbicos, es decir que el usuario se inquieta o tiene actitudes aprensivas hacia la tecnología. La ansiedad percibida, como fenómeno psicológico, ha sido bien investigada en las tres últimas décadas (Beckers; Schmidt, 2001). En general hace referencia a emociones negativas en los estados cognitivos evocados en la interacción real o imaginaria con la tecnología informática.

Las manifestaciones del comportamiento de ansiedad hacia los ordenadores incluyen: 1) evitarlos; 2) precaución excesiva respecto a los mismos; 3) sensaciones de negativa ante ellos; y 4) procurar acortar el tiempo necesario de uso (Bozionelos, 2001). Con el aumento de la penetración de los Pcs el problema de la ansiedad hacia el ordenador ha aumentado (Huang; Liaw, 2005). 


\section{"La ansiedad percibida, como fenómeno psicológico, ha sido bien investigada en las tres últimas décadas"}

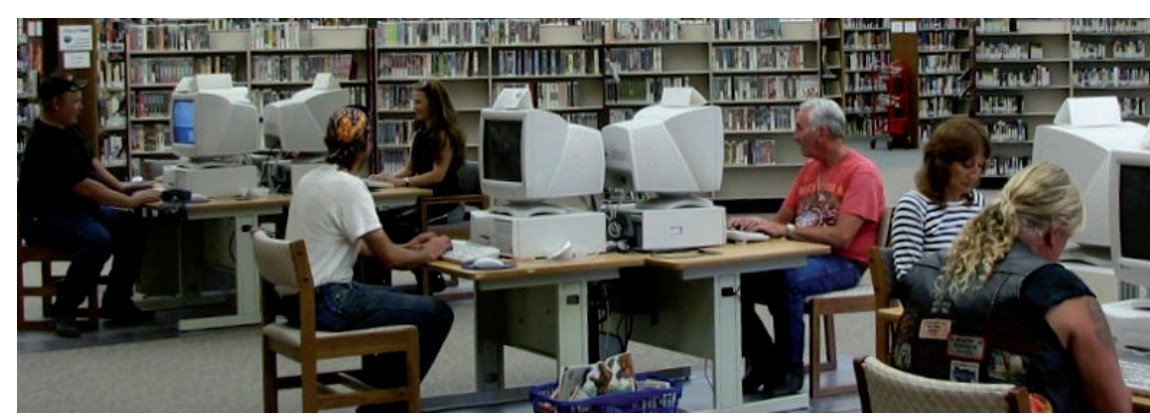

Algunos estudios (Durndell; Haag, 2002; Nash; Moroz, 1997) afirman que la ansiedad percibida tiene relaciones significativas con la satisfacción y la utilidad percibidas. Además la ansiedad hacia el ordenador se confirma como un factor negativo en las actitudes de los usuarios respecto a la facilidad de uso de las tecnologías (Hackbarth; Grover; Yi, 2003).

\section{Eficacia percibida}

La eficacia se refiere a la creencia del individuo en sus capacidades para activar la motivación, los recursos cognitivos, y las líneas de conducta necesarias con el fin de resolver una demanda circunstancial determinada (Wood; Bandura, 1989). La mayoría de los individuos se percibe como capaz de ser más activo y más eficiente, por lo que persiste en sus esfuerzos. Por tanto la eficacia refleja la confianza de un individuo en su capacidad de realizar las acciones requeridas para producir resultados específi$\cos$, lo que incidiría directamente en la decisión de enrolarse en una tarea, en el esfuerzo que ello exigiría y en la persistencia exhibida (Kinzie; Delcourt; Powers, 1994). Este debate acentúa el impacto del estado cognitivo de los usuarios en los resultados obtenidos y la importancia de entender correctamente el sentido de la eficacia.

Por otra parte las influencias ambientales tales como las presiones sociales o los factores circunstanciales únicos (la personalidad, las características demográficas y los comportamientos), propios de las características cognoscitivas y otras de tipo personal se influyen recíprocamente. Bandura (1986) considera la eficacia como la capacidad generativa en la cual lo cognitivo, lo social y el comportamiento deben organizarse en líneas de conducta integradas para servir a innumerables propósitos.

Aquí se menciona la dimensión de lo social como otro elemento más a tener en cuenta. Efectivamente, se ha demostrado que la exposición a las TIC influye en el bienestar psicosocial de los usuarios en un sentido tanto positivo (entusiasmo, experiencias óptimas o flow) como negativo -tecnoestrés- (fatiga informativa, ansiedad) (Salanova, et al., 2004). El tecnoestrés está directamente relacionado con los bajos niveles de autoeficacia vinculada a la tecnología y se ha puesto de manifiesto el poder de las propias creencias acerca de la eficacia como un recurso personal para hacerle frente. Además la teoría cognitiva social ha dado pie para extender el marco hacia la eficacia colectiva, es decir, la creencia grupal compartida sobre las competencias conjuntas encaminadas a organizar y ejecutar las acciones requeridas para producir determinados resultados.

La eficacia colectiva percibida y la autoeficacia tienen funciones similares y operan a través de los mismos procesos psicosociales. Según la teoría social cognitiva las principales fuentes de autoeficacia y de eficacia colectiva son: 1) las experiencias de éxito; 2) la experiencia vicaria -sensaciones y emociones que se viven a través de las experiencias de otras personas-; 3) la persuasión verbal y; 4) los estados emocionales y somáticos. Entre todas estas fuentes de eficacia destacan las experiencias de éxito o dominio, pues el éxito repetido en determinadas tareas aumenta las evaluaciones positivas de eficacia mientras que los fracasos repetidos las disminuyen. Además se ha encontrado evidencia empírica sobre el modelo espiral de la autoeficacia como un círculo vicioso (autoeficacia negativa) y como un círculo virtuoso (autoeficacia positiva) y se ha puesto de manifiesto que las experiencias de éxito son una fuente de autoeficacia positiva (Salanova, et al., 2004).

Estas concepciones teóricas adquieren particular importancia en estos momentos ante el avance de las redes sociales en el contexto de la web 2.0 con un gran potencial para el uso y desarrollo de herramientas colaborativas cuya eficacia está basada en el trabajo cooperativo que facilite el intercambio de información de los equipos de estudio.

\section{"Estas concepciones teóricas adquieren particular importancia en estos momentos ante \\ el avance de las redes sociales en el contexto de} la web 2.0"

De acuerdo con diversos estudios realizados, la eficacia percibida tiene relación positiva con la utilidad y la satisfacción percibidas (Liaw, 2002a, 2002b). 


\section{Satisfacción percibida}

Se refiere a gozar del trabajo en una actividad específica. Las perspectivas de motivación también se han utilizado extensamente para entender el comportamiento individual según lo cual la satisfacción percibida es un tipo de motivación intrínseca que acentúa el placer y la satisfacción inherente derivada de una actividad específica (Vallerand, 1997). Como señalan Salamina, et al (2004) el uso de tecnologías produce también efectos positivos sobre el bienestar psicológico (flow). Flow es un estado psicológico en el cual un individuo se siente cognitivamente eficaz, motivado y feliz. Cuando la persona experimenta este estado se concentra en su actividad y deja atrás pensamientos irrelevantes. Es un estado de "experiencia óptima".

\section{Utilidad percibida}

Se define como el grado en el cual una persona cree que el usar una tecnología aumentará su productividad (Vankatesh, 1999). De acuerdo con la perspectiva del modelo TAM, es un tipo de expectativa respecto a los resultados que conlleva la intención individual de utilizar tecnologías y sistemas de información reales. Es una clase de motivación extrínseca que describe la ejecución de un comportamiento destinado a alcanzar una meta específica o determinada recompensa (Deci; Ryan, 1987) y provoca satisfacción por lo que tiene un efecto positivo y constituye uno de los factores que influye con mayor peso sobre la intención de utilizar tecnologías de la información.

Talja, Savolainen y Maula (2005) realizaron un estudio comparativo cualitativo a través de cuatro dominios científicos (enfermería, literatura y estudios culturales, historia y biología ambiental), explorando el uso y la utilidad percibida en las listas de discusión en las que participaban los estudiantes y su relación con determinados ele- mentos, entre ellos: los métodos de búsqueda de información; los patrones de colaboración; la localización de la información; la proximidad física de colegas de la misma especialidad; la estructura del campo temático; el deseo de compartir la información en foros de discusión; los criterios de relevancia; el grado de dispersión dentro de un campo, y la preferencia por determinados soportes de la información (libro vs. artículo).

Los resultados de este estudio demostraron el papel diferenciado que adquiere la comunicación mediada por ordenador a través de los diversos campos temáticos, y contribuyeron a la comprensión de los factores subyacentes presentes en las redes sociales. Los biólogos ambientales y los científicos del ámbito de la enfermería encontraron que la participación en las listas de discusión era de poco valor para ellos. Ellos confían más en la revisión sistemática de la literatura de la especialidad y en sus colaboradores locales como fuentes de información. En cambio los historiadores, los literatos y los eruditos en estudios culturales comprobaron que son provechosas para la revisión de la literatura y para el desarrollo del campo temático. Para los humanistas el hecho de poder compartir interpretaciones de documentos con otros colegas es una práctica central dentro del proceso de la investigación ya que actúan a menudo como "filtros de autores" y orientan la búsqueda de información entre sus pares.

Los estudios comparativos sirven para mejorar los diseños de los sistemas de información de manera que puedan resolver con acierto las necesidades de esas comunidades.

\section{Algunas aproximaciones al estudio de la aceptación y uso de la tecnología web}

Puesto que internet y la web son extensamente populares, el uso de estas tecnologías se ha extendido rápidamente a muy diversos aspectos de nuestra actividad y de nuestra vida. Al igual que ocurre con la aceptación del uso de los ordenadores en general, muchos estudios indican que el éxito de la utilización

\section{"El éxito de la utilización de las herramientas tecnológicas de la web depende en gran parte de las actitudes de los usuarios hacia la misma"}

de las herramientas tecnológicas de la web depende en gran parte de las actitudes de los usuarios hacia la misma. (Johnson; Hignite, 2000; Lederer, et al., 2000; Liaw, 2002a, 2002b; Moon; Kim, 2001).

Aunque el concepto "actitud hacia los ordenadores" no es una definición universalmente aceptada, es un determinante crítico de uso y aceptación de las TIC. Una herramienta de análisis que persiga entender cómo los usuarios emplean la web, deberá permitir examinar claramente la correlación entre la ansiedad y la satisfacción percibidas, así como la correlación entre la ansiedad y la utilidad percibidas.

Encontramos ejemplos de aplicación de TAM en muy diferentes ámbitos de internet y de la www, desde la banca (Lai; Li, 2005) y el comercio electrónico (Kim; Lee; Law, 2007) hasta el e-learning (Saadé; Kira, 2007) o el diseño de interfaces (Hasan; Ahmed, 2007; Lee; Kang; Kim, 2007). En relación a las herramientas de búsqueda en la web, los estudios realizados (Liaw, et al., 2006; Liaw; Huang, 2003 y 2004) demuestran que el disfrute $y$ la facilidad de empleo percibidos pueden influir sobre la utilidad percibida de los buscadores web. Las teorías principales del modelo de investigación de Liaw y Huang deri- 


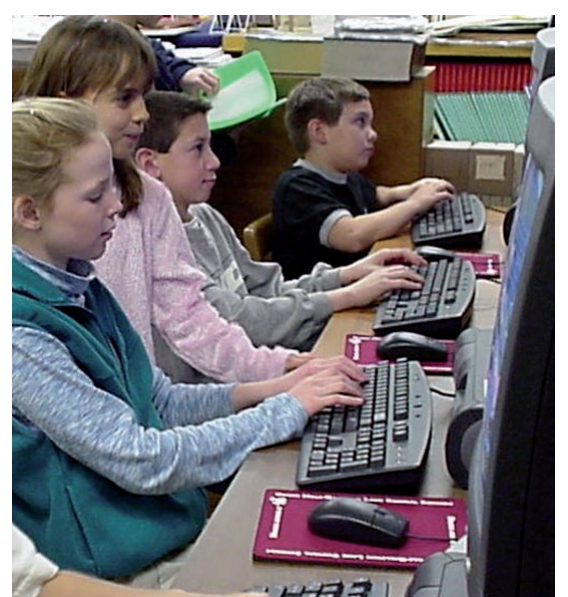

van del TAM pero evolucionan hacia los 3-TUM (three-Tier Use Model), que tienen en cuenta tres factores relevantes para predecir el comportamiento individual al utilizar la tecnología con un propósito particular (por ejemplo como una herramienta de aprendizaje o de recuperación de información): la calidad del sistema y la experiencia individual; el factor afectivo y cognitivo; y el componente de intención.

Por tanto resulta evidente que para conseguir logros y éxitos, la tecnología, los sistemas y las aplicaciones, -en general, y en el entorno de la web en particular- han de atender a las expectativas y percepciones de los usuarios. En este sentido se habla de evaluación centrada en el usuario cuando se aplican, diseñan y/o desarrollan modelos y metodologías en un esfuerzo por optimizar (en el plano tecnológico y subjetivo) las interacciones hombre-máquina.

\section{Reflexiones finales}

Ante el término usuario habitualmente se asume que éste se trata de un consumidor (real o potencial) de información, soslayando que se está frente a un individuo con una personalidad definida, con su carga emocional, sus valores culturales, su lengua, sus costumbres. En suma, un ser humano condicionado por el tiempo y el espacio en el que le toca vivir. Las emociones de cada persona tienen una gran repercu- sión en su forma de pensar y en las acciones que realiza.

La evolución de la humanidad nos ubica hoy en la era de las TIC. Estamos inmersos en una nueva cultura, con nuevos valores, nuevos requerimientos de capacidades y destrezas, nuevas instituciones, nuevas profesiones, donde la información es acumulable, donde puede ser almacenada y reproducida para volver a ser reutilizada, produciendo más información. Sin embargo todo parece indicar que no importa cuan sofisticada y de cuánto es capaz la tecnología, su puesta en práctica eficaz depende de los usuarios que tienen una actitud positiva hacia ella (Culpan, 1995).

La utilización de las TIC ofrece a los usuarios la oportunidad de realizar o desarrollar ciertas acciones de una manera más independiente, más eficiente. Estamos viviendo un tiempo de cambio total en la cultura tecnológica, nos situamos en la etapa de las redes sociales y la web 2.0, que dejó atrás a los sitios web estáticos limitados a expertos para dar paso a portales controlados por los usuarios. En este escenario centrado en el usuario, la disponibilidad y la actitud de los individuos implicados en el uso de las tecnologías es un factor crítico de éxito en los resultados que se obtienen.

\section{Bibliografía}

Al-Khaldi, M. A.; Al-Jabri, I. M. "The relationship of attitudes to computer utilization: New evidence from a developing nation". En: Computers in human behavior, 1998, v. 14, n. 1, pp. 23-42

Bagozzi, R. P.; Davis, F. D.; Warshaw, P. R. "Development and test of a theory of technological learning and usage". En: Human relations, 1992, v. 45, n. 7, pp. 660-686.

Bandura, A. Social foundations of thought and action. Englewood Cliffs, NJ: Prentice-Hall, 1986.

Beckers, J. J.; Schmidt, H. G. "The structure of computer anxiety: a six-factor model". En: Computers in human behaviors, 2001, v. 17, n. 1, pp. 35-49.

Bozionelos, N. "Computer anxiety: relationship with computer experience and prevalence". En Computers in human behavior, 2001, v. 17, n. 2, pp. 213-224.

Compeau, D. R.; Higgins, C. A. "Application of social cognitive theory to training for computer skills". En: Information systems research, 1995, v. 6, n. 2, pp. 118-143.

Culpan, O. "Attitudes of end-users towards information technology in manufacturing and service industries". En: Information \& management, 1995, v. 28, n. 33, pp. 167-176.

Davis, F. D. "User acceptance of information technology: system characteristics, user perception and behavioral impacts". En: International journal of man-machine studies, 1994, v. 38, n. 2, pp. 475-487.

Davis, S.; Wiedenbeck, S. "The mediating effects of intrinsic motivation, ease of se and usefulness perceptions on performance in first-time and subsequent computer users". En: Interacting with computers, 2001, n. 13, pp. 549-580.

Deci, E. L.; Ryan, R. M. "The support of autonomy and the control of behaviour". En: Journal of personality and social psychology, 1987, v. 53, n. 6, pp. 1.024-1.037.

Durndell, A.; Haag, Z. "Computer self efficacy, computer anxiety, attitudes toward the internet and reported experience with the internet, by gender, in an East European sample". En: Computers in human behavior, 2002, v. 18, n. 5, pp. 521-535.

Gressard, C. P.; Loyd, B. H. "Validation studies of a new computer attitude scale". En: Association of Educational Data Systems journal, 1986, v. 18 , n. 4 , pp. 295-301.

Hackbarth, G.; Grover, V.; Yi, M. Y. "Computer playfulness and anxiety: positive and negative mediators of the system experience effect on perceived ease of use". En: Information management, 2003, v. 40, n. 3, pp. 221-232.

Hasan, B.; Ahmed, M. U. "Effects of interface style on user perceptions and behavioral intention to use computer. En: Computers in human behavior, 2007, v. 23, pp. 3.025-3.037

Hebert, M.; Benbasat, I. "Adopting information technology in hospitals: trelationship between attitudes/expectations and behaviour". En: Hospital \& health services administration, 1994, v. 39, n. 3, pp. 369-383.

Huang, H. M; Liaw, S. S. "Exploring users' attitudes and intentions toward the web as a survey tool". En: Computers in human behavior, 2005, v. 21, n. 5 , pp. $729-743$.

Johnson, R. A.; Hignite, M. A. "Student usage of the world wide web: a comparative study". En: Journal of computer information systems, 2000, v. 40, n. 4, pp. 93-97.

Kay, R. "An exploration of theoretical and practical foundations for assessing attitudes toward computers: the Computer Attitude Measure (CAM)". En: Computers in human behavior, 1993, v. 9, n. 4, pp. 371-386.

Kim, T. G.; Lee, J. H.; Law, R. "An empirical examination of the acceptance behaviour of hotel front office systems: an extended technology acceptance model". En: Tourism management, 2007 (en prensa).

Kinzie, M. B.; Delcourt, M. A. B.; Powers, S. M. "Computer technologies: attitudes and selfefficacy across undergraduate disciplines". En: Research in higher education, 1994, v. 35, n. 6, pp. 745-768. 
Lai, V. S.; Li, H. "Technology acceptance model for internet banking: an invariance analysis". En: Information and management, 2005, v. 42, n. 2 , pp. 373-386.

Lee, K. C.; Kang, I.; Kim, J. S. "Exploring the user interface of negotiation support systems from the user acceptance perspective". En: Computers in human behavior, 2007, v. 23, pp. 220-239.

Liaw, S. S. "An internet survey for perceptions of computers and the world wide web: relationship, prediction, and diference". En: Computers in human behavior, 2002a, v. 18, n. 1, pp. 17-35.

Liaw, S. S. "Understanding user perceptions of world-wide web environments". En: Journal of computer assisted learning, 2002b, v. 18, n. 2, pp. 139-150.

Liaw, S. S.; Huang, H. M. "Information retrieval from the world wide web: a user-focused approach based on individual experience with search engines". En: Computers in human behavior, 2004, v. 22, n. 3, pp. 501-517.

Liaw, S. S., et al. "Attitudes toward search engines as a learning assisted tool: approach of Liaw and Huang's research model". En: computers in human behavior, 2006, v. 22, n. 2, pp. 177-190.

Loyd, B.; Gressard, C. "Reliability and factorial validity of computer attitude scales". En: Educational and psychological measurement, 1984, v. 44 , n. 2, pp. 501-505.
Nash, J. B., Moroz, P. A. "An examination of the factor structures of the computer attitude scale". En: Journal of educational computing research, 1997, v. 17 , n. 4, pp. 341-356.

Popovich, P. M.; Hyde, L. R.; Zakrajsek, T. "The development of the attitudes toward computer usage scale". En: Educational and psychological measurement, 1987, v. 47, n. 1, pp. 261-269.

Saadé, R. G.; Kira, D. "Mediating the impact of technology usage on perceived ease of use by anxiety". En: Computers \& education, 2007, v. 49 , n. 4, pp. $1.189-1.204$

Salanova, M., et al. "Autoeficacia y tecnologías de la información y comunicación”. En: Nuevos horizontes en la investigación sobre autoeficacia. Castellón: Colección Psique, 2004, n. 8, pp. 91-98. Consultado en: 17-09-07.

https://bscw.uji.es/pub/bscw.cgi/d316654

Talja, S.; Savolainen, R.; Maula, H. "Field differences in the use and perceived usefulness of scholarly mailing lists". En: Information research, 2005, v. 10, n. 1, paper 200. Consultado en: 17-09-07.

http://InformationR.net/ir/10-1/paper200.html

Vankatesh, V. "Creation of favorable user perceptions: exploring the role of intrinsic motivation". En: MIS quarterly, 1999, v. 23, n. 2, pp. 239-260.

Vallerand, R. J. "Toward a hierarchical model of intrinsic and extrinsic motivation". En: Advances in experimental social psychology, 1997, n. 27, pp. 271-360

Wood, R. E.; Bandura, A. "Impact of conceptions of ability on self-regulatory mechanisms and complex decision making". En: Journal of perceived social psychology, 1989, v. 56, n. 3, pp. 407-415.

María-Dolores Olvera-Lobo, Departamento de Biblioteconomía y Documentación, Facultad de Comunicación y Documentación, Universidad de Granada, Campus Cartuja, Colegio Máximo 18071, Granada. molvera@ugr.es

\section{Belarmina Benítez-de-Vendrell,} Departamento de Bibliotecología, Facultad de Humanidades y Ciencias Sociales, Universidad Nacional de Misiones, Posadas, Misiones, Argentina.

BBVendrell@arnet.com.ar

\section{nature.com}

es ciencia

nature.com
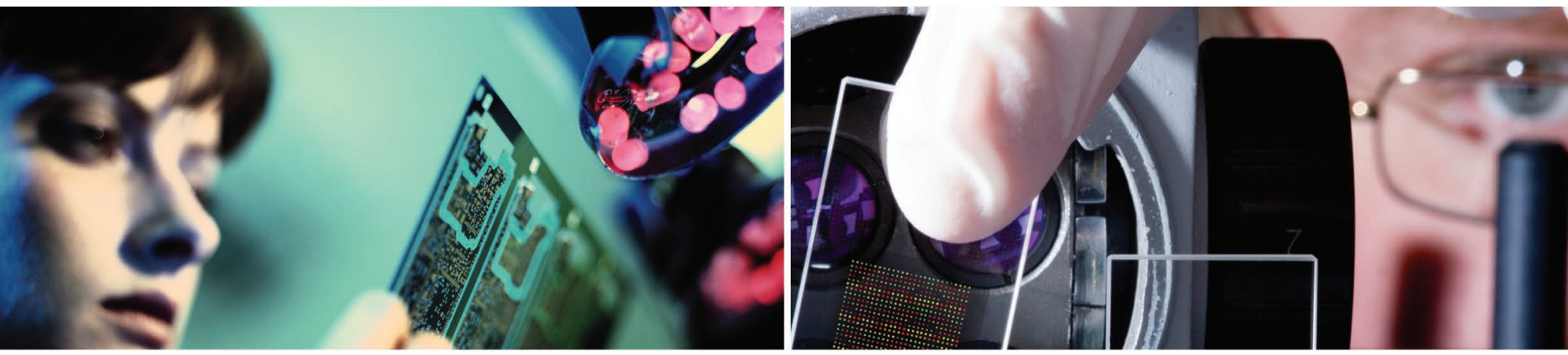

\section{Todo está en nuestro nature.com}

Consiga su acceso a nature.com con una Licencia de Acceso y elija entre las revistas de investigación científica y medica de más alta calidad. Desde la compartición de documentos hasta la evaluación de artículos por expertos, nature.com ofrece numerosas aplicaciones emprendedoras en Web 2.0, entre las que encontrará

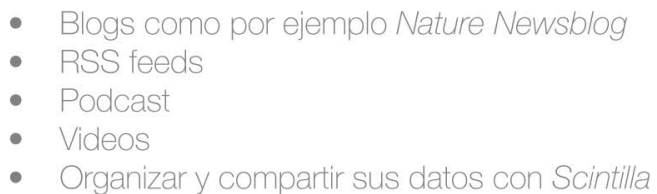

- Referencia de documentos con Connotea

- Redes sociales en Nature Network

- Nature Precedings, un foro que le permite compartir su trabajos de investigación antes de su publicación 